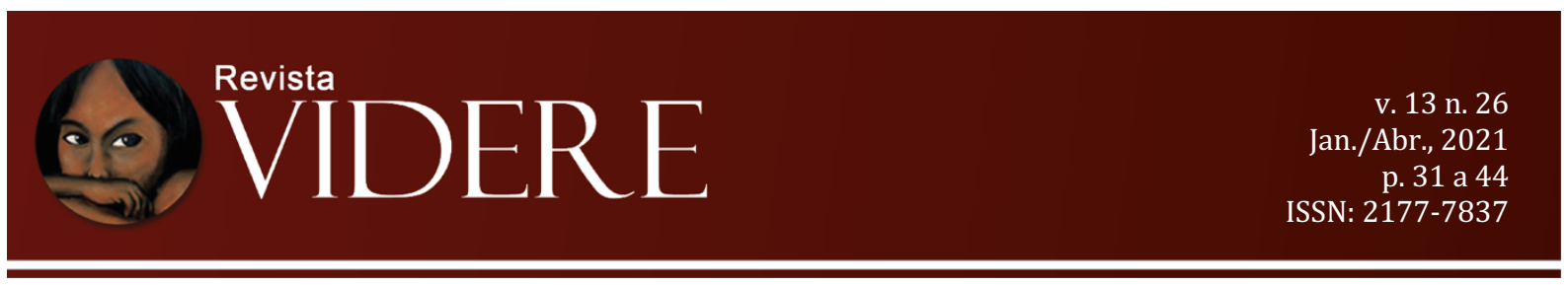

\title{
LUCHAS INDÍGENAS, EN LOS ÚLTIMOS AÑOS, EN LA SIERRA NORTE DE PUEBLA: EL USO DEL DERECHO
}

\author{
INDIGENOUS STRUGGLES, IN THE RECENT YEARS, \\ IN THE SIERRA NORTE OF PUEBLA: THE USE OF RIGHT \\ LUTAS INDÍGENAS, NOS ÚLTIMOS ANOS, \\ NA SIERRA NORTE DE PUEBLA: O USO DO DIREITO
}

Jesús Antonio de la Torre Rangel Profesor-Investigador de la Universidad Autónoma de Aguascalientes, México

E-mail: beastvtr@prodigy.net.mx OrcidID: orcid.org/0000-0001-5664-0208http

\begin{abstract}
Resumo: La Sierra Norte de Puebla, México, es territorio mayoritariamente de cultura indígena: nahua y totonaca. En los últimos años se ha dado una organización ejemplar, con el objeto de defender sus derechos como pueblos indígenas y para resistir en la conservación de su territorio, por la amenaza de los megaproyectos hidroeléctricos y mineros. Aquí se narra parte de esas luchas.
\end{abstract}

Palabras clave: derechos indígenas, insurgencias jurídicas, resistencia.

Abstract: The Sierra Norte of Puebla, México, is mostly indigenous culture territory: nahua and totonaca. In the last years there has been an exemplary organization, in order to defend his rights as indigenous peoples and to resist in conservation of their territory, because of the threat of hydroelectric and mining megaprojects. Part of these struggles are narrated here.

Keywords: indigenous rights, legal insurgencies, resistance.

Resumo: A Sierra Norte de Puebla, México, é principalmente território de cultura indígena: nahua e totonaca. Nos últimos anos, tem havido uma organização exemplar, a fim de defender seus direitos como povos indígenas e resistir na conservação de seu território, por causa da ameaça de megaprojetos hidrelétricos e mineradores. Parte dessas lutas são narradas aqui.

Palavras-chave: direitos indígenas, insurgências legais, resistência. 


\section{Introducción}

Siguiendo algunas enseñanzas de Dussel, en cuanto a su modo de entender los "sujetos de la historia", Gabriel Hernández, refiriéndose al movimiento indígena en los últimos años en la Sierra Norte de Puebla, dice que se trata de un proceso de toma de conciencia crítica, "que deviene acción colectiva, articulada con una identidad específica, la identidad étnica, que a su vez, por los factores estructurales de dominación... se ha configurado en una etnicidad. Ésta última se transforma paulatinamente en una columna vertebral que orienta la praxis política" (Hernández García, 2012, págs. 248-249). Precisamente por esa conciencia crítica de las causas que lo niegan, tiene una subjetividad liberadora y lleva a cabo políticas en ese sentido el movimiento indígena.

Hernández nos narra procesos sociales contrahegemónicos de nuevos actores que producen un movimiento indígena de totonacas y nahuas en la Sierra Norte de Puebla; precisamente en Totonacam que es el nombre con el que se conoce el territorio habitado históricamente por el pueblo totonaca, que sufrió penetraciones mexicas (cultura nahua) en determinadas porciones, como en Quetzalan y Yehualichan; precisamente en la Sierra Norte de Puebla se encuentra a la orilla de ese histórico territorio, en un macizo montañoso.

En la Sierra Norte de Puebla, México, se están dando procesos de lucha muy importantes contra megaproyectos hidroeléctricos y mineros de diverso tipo, por indígenas totonacas y nahuas asociados con mestizos, combinando diversas estrategias político-jurídicas. En esos procesos de lucha el Derecho juega un papel muy importante. Podemos decir que es derecho que nace del pueblo o derecho insurgente, en dos sentidos: uno, como normatividad autónoma, producida por las decisiones de las asambleas y por sus prácticas comunitarias; y dos, la apropiación que hacen de las normas producidas por el Estado que les reconocen derechos y las utilizan en su beneficio, legitimando sus luchas.

En este artículo se hablará un poco de estas luchas de resistencia.

\section{Tierra y Territorio en la Sierra Norte de Puebla}

La cultura totonaca tiene una cosmovisión de la tierra que la considera como una divinidad dual, como Madre y Padre, “desempeñando un papel determinante en la vida de este pueblo, ya que les recuerda su doble origen: como seres humanos y como pueblo." (Rodríguez, 2020, p. 32). 
El territorio implica a la tierra, a el agua, a la vida misma, por eso se hace necesario defenderlo. Dice Natalia Rodríguez que el territorio aparece en el pensamiento del pueblo totonaco, según expresa Marisol de la Cadena, como "un complejo que tiene lugar relacionalmente", "ocurre en el tiempo y en el espacio, relacional y simultáneamente, que impide la separación de gente y territorio, porque los dos son al mismo tiempo, y son los dos juntos." (Rodríguez, 2020, p. 35).

A pesar de tratarse de territorio indígena, una gran cantidad de tierra está privatizada y es escaza: "no existe, en la mayor parte de la sierra, un régimen de propiedad colectiva de la tierra, por lo que los pequeños propietarios constituyen a la mayoría de los campesinos." (Hernández, 2012, p. 40). El crecimiento demográfico reduce el tamaño de las parcelas, pues se dividen entre cada vez más propietarios, y por lo mismo, más pequeños.

El hecho de que, siendo territorio indígena, prevalezca en la Sierra Norte de Puebla la tenencia de la tierra como propiedad privada, es un hecho extraño en México. La inmensa mayoría de los pueblos indígenas a lo largo y ancho del País, conservan la tenencia de la tierra como "comunidad indígena" o como "ejidos", en ambos supuestos de manera comunitaria. La explicación de este hecho insólito, la encontramos en que los pueblos indígenas de la Sierra Norte de Puebla, aceptan la privatización de la tierra como estrategia de defensa de su territorio. En el siglo XIX, en que los gobiernos liberales promovieron las leyes de desamortización de la tierra, que implicaba el despojo de éstas a los pueblos y comunidades indígenas, los totonacas y nahuas de la Sierra Norte de Puebla, deciden luchar al lado de los liberales en la guerra contra los conservadores, aceptando la privatización de la tierra, pero conservándola y conservando también el territorio. Siguiendo a Korinta Maldonado Goti, Natalia Rodríguez concluye: "La estrategia por parte de los líderes liberales de la Sierra, por medio de la cual, se habría permitido la privatización de la tierra comunal, pero al mismo tiempo, logrando la conservación de gran parte de las mismas en manos de los pueblos indios, sería una explicación al porqué muchas de las tierras fueron conservadas por los habitantes originarios de la Sierra, en forma de pequeña sociedad." (Rodríguez, 2020, p. 48).

En los años ochenta del siglo pasado, surgen y se consolidan varias organizaciones indígenas en la región. Pero antes ya, en los años setentas, surgió la Unión Campesina Independiente (UCI), una experiencia de lucha por la tierra, por el reparto agrario, bastante combativa. Pero fue durante reprimida. Incluso Antorcha Campesina (AC), se creó por esos años "para mediatizar las demandas sociales y para facilitar la represión del Estado" (Hernández, 2012, p. 56); con un discurso de extrema izquierda, sin embargo articulada al 
Partido Revolucionario Institucional (PRI), frenaba a los movimientos campesinos y sus demandas.

Con la UCI desarticulada y con presos políticos, "poco a poco las reivindicaciones agrarias dejaron de ser la demanda central y cada vez más los campesinos indígenas empezaron a organizarse, obligados por las circunstancias, como pequeñas unidades de producción agrícola. En distintas regiones, las organizaciones campesinas de corte productivista se convirtieron en una alternativa para canalizar el descontento social que existía en las zonas rurales. Estaban orientadas a impulsar proyectos productivos propios y controlar también el mercado.” (Hernández, 2012, p. 60-61).

\section{Teología de la Liberación y Teología India, en el trasfondo de la conciencia crítica}

La Arquidiócesis de Puebla, por la acción de varios agentes en la Sierra Norte de Puebla, impulsó el movimiento por el reconocimiento y la defensa de los derechos indígenas, Inspirados, en principio, en la Teología de la Liberación, teniendo al pobre como sujeto de la historia, se impulsan las Comunidades Eclesiales de Base (CEBS). Sin embargo, se da una transición de la Teología de la Liberación a la Teología India, así cambia el sujeto político: la segunda tomó para si los principios político-críticos de la primera, "pero trató de ir más allá, tratando de hacer emerger la visión que sobre la liberación tienen los pueblos nahuas y totonacas de la sierra." (Hernández, 2012, p. 83).

Sacerdotes y religiosas de la región llevaron a cabo un proceso de formación de cuadros, conocidos como catequistas para potenciar las comunidades de base. Leían la Biblia, pero también instrumentos jurídicos referentes a los derechos de los pueblos indígenas, como la propia Constitución y en Convenio 169 de la Organización Internacional del Trabajo (OIT). Destaca la labor de sacerdotes como los padres Mario Pérez, Juan Ramírez y Salvador Sotero; y de religiosas como las misioneras Carmelitas de Santa Teresita del Niño Jesús y las misioneras Guadalupanas del Espíritu Santo.

\section{La Organización Independiente Totonaca}

En Huehuetla se va a iniciar un movimiento de liberación indígena, con la Organización Independiente Totonaca (OIT), que tiene como antecedente directo las Comunidades Eclesiales de Base, gestándose en los años 80. Gabriel Hernández, expresa que: 
En los orígenes de esta organización jugó un papel importante la vinculación ritual con la tierra que existe en los pueblos, expresada en lo que ellos mismos llaman la espiritualidad de la tierra. La promoción de la Teología Indígena contribuyó también a fortalecer el proceso organizativo (Hernández, 2012, p. 90).

Luego cita un documento interno de la OIT:

Quien nos acompañó en este proceso fueron algunas misioneras y misioneros cristianos, algunos profesionistas, ellos ayudaron. Con velitas leíamos, andábamos en las noches y con lluvias, pero no importaba porque nos alumbra una luz que nos guiaba por los caminos, hacíamos en las noches reuniones en nuestras casas para alimentar esa luz y junto a nuestro libro sagrado llevábamos también la Constitución, y de esta manera nacimos como organización y como gobierno [...] (Hernández, 2012, p. 91).

En 1992 se definen los "principios rectores" de la OIT, que son los siguientes:

1. Se reconoce al pueblo de Huehuetla como pueblo totonaco y por ende se reconoce su derecho a la libre autodeterminación.

2. En el centro de la organización se encuentra Dios Padre.

3. La organización dará prioridad antes que nada a todo lo totonaco.

4. Se plantea la organización como defensa y proyecto de liberación.

5. La organización se proclama por los movimientos sociales que estén a favor de un proyecto de liberación.

6. La organización se proclama por el derecho de la mujer totonaca a participar con equidad.

7. La OIT es una organización popular y a ella podrán pertenecer todos los que luchen contra la opresión y por los derechos humanos (Hernández, 2012, p. 92).

La estructura organizativa de la OIT, se hizo de este modo:

1. La directiva de la OIT como representante de la organización social será elegida por medio de la votación y tiene como obligación, convocar a asambleas. 2. El consejo de la organización integrado por la directiva y los coordinadores de cada comisión, se reunirá cada mes para mantener un buen funcionamiento. 3. OIT se reunirá cada mes por lo menos y las asambleas generales de la organización serán cada cuatro meses. 4. La última palabra la tiene la asamblea general. 5. La representación de las comunidades se hará por medio de comisionados quienes funcionarán como puente entre las comunidades y la directiva. (Hernández, 2012, p. 92 y 93).

En lo interno se organizó en llamados "departamentos":

1. Departamento de comercialización del café.

2. Departamento de comercialización de pimienta.

3. Departamento de tortillería y masa.

4. Departamento de productos de artesanía.

5. Departamento de farmacia de medicina tradicional. 
6. Departamento de tienda de abasto de productos populares.

7. Departamento de caja popular.

8. Departamento de microempresas comunitarias en escuelas.

9. Departamento del área educativa.

La Organización Independiente Totonaca, como dice Gabriel Hernández, interpela a la "sociedad otra" en estos términos, al exponer como es su organización:

Los indígenas totonacos que padecemos por estas tierras del Totonacapan de la Sierra Norte del Estado de Puebla, desde este lunarcito de patria, que es Huehuetla, nos dirigimos a ustedes con la finalidad de darles a conocer nuestro caminar en esto de querer seguir siendo indígenas, con nuestra lengua, con nuestra vestimenta, tradiciones y costumbres. Pero por favor, no nos vean como hombres que quieren seguir siendo miserables, pobres, analfabetas y "mexicanos de cuarta", como nos mira la sociedad occidental clásica, no. Queremos seguir siendo indígenas totonacos porque queremos convencerlos que ustedes sean mexicanos verdaderos, queremos que ustedes se parezcan a nosotros y compartir con ustedes nuestra manera de ver el mundo en ese sentido, y no solo eso, sino su ciencia y su técnica, queremos retomar nuestro mundo desde nuestras raíces. Con todo ello, nos atrevemos a hacerles una propuesta de sociedad y de modo de vida, de cara a la sociedad occidental y a otros mundos: la sociedad comunitaria. ¡Pero no se espanten! No es socialismo ni comunismo, es simplemente comunidad, es la misma propuesta de nuestros abuelos y padres primeros (lakttajni y laknanajni), se trata de revalorar nuestra cultura madre, la de la hermandad, la de la solidaridad verdadera, la de la comunidad (Hernández, 2012, p. 93 y 94).

La OIT expone sus insurgencias jurídicas, esto es, sus normas comunitarias con prácticas como la "mano vuelta", y el ejercicio de los cargos de gobierno como servicio, su modo de entender el "mandar obedeciendo":

Nuestro modo de vida sigue siendo comunitario, muy a pesar de que ha penetrado por todos los medios a su alcance el mundo de lo individual, de la mezquindad y de la religión del dinero, donde se tiene por dios al dinero. Nuestro modo de vida sigue siendo comunitario porque seguimos conservando las costumbres de nuestros abuelos y padres primeros, bajo la base del trabajo por servicio para obras de comunidad. Seguimos siendo comunidad y con ello totonacos porque aún conservamos el trabajo de "mano vuelta", que permite realizar los trabajos de la producción mediante la ayuda mutua, cuando hay necesidad, trabajamos en el rancho de un hermano porque él también nos apoyará cuando lo necesitemos. Nuestro modo sigue siendo comunitario porque en las fiestas, reuniones, costumbres y tradiciones compartimos lo que tenemos, lo que pensamos, los cargos y representación de gobierno comunitario son en función de todo ello. Seguimos siendo comunidad porque hacemos elecciones en comunidad y no a las escondidas o en secreto. La comunidad todo lo vigila, todo lo fiscaliza, lo califica y todo lo mide en función del trabajo de comunidad, a tal grado que los cargos en el gobierno comunitario están en función de la demostración de una conducción apegada a las normas éticas de la comunidad. De tal suerte que nuestra propuesta es también una propuesta ética. La comunidad es una propuesta ética porque se vigila el deber ser, según las normas de la comunidad, la conducción de los totonacos y totonacas en la comunidad tiene gran apego a 
principios, la mayoría de ellos no escritos, pero vigilados celosamente a tal grado que los hombres y mujeres se deben cuidar de no violarlos (Hernández, 2012, p. 95).

El gobierno totonaco fue una realidad en el municipio de Huehuetla. La Organización Independiente Totonaca se alió políticamente al Partido de la Revolución Democrática (PRD), y ejerció el poder en tres trienios, en los periodos: 1990-1993, 1993-1996 y 1996-1999. A este período de nueve años se le conoce como "gobierno indígena" o "gobierno totonaco". Esta administración del Ayuntamiento de Huehuetla, enfrentó condiciones difíciles, entre otras, que carecían de experiencia en la administración pública, pues los indígenas gobernaban por primera vez el municipio de Huehuetla; además les habían dejado la presidencia municipal desmantelada, sin recursos. Sin embargo, a pesar de las dificultades en esos años de gobierno, la experiencia fue buena porque permitió a las comunidades indígenas avanzar en organización y conciencia sobre sus derechos políticos, sociales y culturales.

Este gobierno totonaco produjo derecho propio innovador, derecho insurgente, ya que elaboró un "Bando de Policía y Buen Gobierno", en el que:

[...] introduce los derechos indígenas establecidos en el Convenio 169 de la Organización Internacional del Trabajo y el actual artículo 4to constitucional que, por cierto, la Constitución estatal todavía, a estas alturas, no ha incorporado. Por ello, Huehuetla es un ejemplo a seguir por los pueblos de otros municipios que quieren votar este 8 de noviembre a favor de candidatos que manden obedeciendo" (Hernández, 2012, p. 95).

Es importante decir, también, que la organización social tradicional totonaca, esto es, los servicios comunitarios que forman parte del sistema de cargos cívicos-religiosos se trasladaron a la estructura del gobierno municipal, como topiles, fiscales, semaneros y mayordomos (Hernández García, Op. Cit., p. 118)

Al terminar los periodos de gobierno indígena, los mestizos regresaron al poder municipal y tratan de ir en contra del proyecto indígena. Se dedicaron a atacar sistemáticamente el "Centro de Estudios Superiores Indígenas Kgoyom" (CESIK), un bachillerato alternativo con un modelo de educación intercultural que tomó la OIT. El hostigamiento llegó al colmo, al asesinar a la abogada Griselda Tirado Evangelio, fundadora del CESIK y asesora de la OIT, el 6 de agosto de 2003.

No obstante tantas dificultades, como amenazas, represión, difamaciones y hostigamiento sistemático, la Organización Independiente Totonaca "se ha mantenido con relativa vitalidad.” (Hernández García, Op. Cit., p. 114). 


\section{Organización Indígena Independiente Ahuacatleca}

Es importante mencionar la Organización Indígena Independiente Ahuacatleca (OIIA), formada por nahuas, totonacos y mestizos, que le interesa la lucha por la justicia social. Se dio a conocer públicamente en el municipio de Ahuacatlán, el 12 de octubre de 1992. Igual que otras organizaciones de la región, la OIIA, comenzó siendo grupo de Comunidades Eclesiales de Base. "La OIIA ha tenido una destacada participación en las movilizaciones y en las acciones por la defensa de la agrodiversidad y en el reconocimiento de los derechos colectivos de los pueblos indígenas a nivel estatal."

\section{Unidad Indígena Totonaca Náhuatl (UNITONA)}

En el año 2000, después de una reflexión integral sobre la situación regional, incluidos los derechos indígenas, la Organización Independiente Totonaca, la Organización Indígena Independiente Ahuacatleca y la Esperanza de los Pobres, decidieron formar, para trabajar unidas, la Unidad Indígena Totonaca Náhuatl (UNITONA). Empezaron con un trabajo organizativo "más porductivista que de acción política" (Hernández, 2012, p. 143); pero para junio de 2001, en un acto público en Cuetzalan, "se planteaba recorrer el camino de la autodeterminación de los pueblos en la autonomía colectiva" (Hernández, 2012, p. 147); implica recuperación de la cultura y la justicia y respeto para los pueblos indios.

La UNITONA fue avanzando en sus objetivos, consolidándose la organización. Su lucha fue cada vez más importante, en lo que se refiere a los derechos de los pueblos indígenas. Su capacidad de convocatoria creció, hasta lograr el "Encuentro de Naciones Indígenas de México", celebrado en Huehuetla del 10 al 12 de octubre de 2003, en el que estuvieron presentes representantes de varios pueblos, de muchas comunidades y buen número de organizaciones indígenas. La Declaratoria del Encuentro es un documento que expresa la conciencia de los indígenas en cuanto a sus derechos de autonomía. Por su importancia la transcribimos:

La palabra es el medio de comunicación entre los pueblos indígenas y representa el respeto, la honestidad y la justicia, hoy decimos nuestra palabra: Por acuerdo de la $10^{\mathrm{a}}$ asamblea del Congreso Nacional Indígena, región Centro-Pacífico, realizada en la comunidad indígena de Bancos San Hipólito, territorio wixarika de Jalisco, los pueblos, comunidades y organizaciones indígenas nos reunimos en el Encuentro de naciones indígenas de México los días 10, 11 y 12 de octubre del 2003, en el municipio indígena totonaca de Huehuetla, de la Sierra Norte de Puebla, para analizar y reflexionar lo siguiente: 


\section{Considerando que:}

-El territorio es el espacio en donde los pueblos indígenas desarrollamos nuestra vida y recreamos nuestra cultura. Cada que se atenta en contra de estos territorios se atenta directamente contra la existencia de nuestros pueblos.

-Las actuales políticas del gobierno están generando procesos que fragmentan estos territorios de diversas maneras, como son los planes de explotación de los recursos naturales por empresas privadas, con capital tanto nacional como extranjero, la introducción de cultivos y plantas ajenas a la región y semillas transgénicas, no solo son un cambio de cultivo sino una agresión contra la tierra, la salud, el medio ambiente y nuestras culturas.

-De la misma manera los conflictos por territorios se agudizan por las políticas del gobierno que violentan los derechos agrarios. El PROCEDE y el PROCECOM en lugar de brindar certeza jurídica a los campesinos, han sido utilizados para enfrentarlos. Ante esto, el gobierno presenta como única alternativa la privatización de las tierras.

- Los templos y centros ceremoniales de nuestros antepasados son la infraestructura para desarrollar los conocimientos y los saberes de nuestros pueblos indios. Nuestra espiritualidad se funda en la relación que los pueblos indios tenemos con la madre tierra, por lo que la respetamos y la veneramos. - Los pueblos indígenas seguimos en el proceso de construcción de la autonomía de hecho, a partir del fortalecimiento de nuestras estructuras comunitarias para la reconstitución integral de nuestros pueblos. Sin embargo, nos enfrentamos a las políticas gubernamentales que no reconocen nuestros derechos como pueblos. En lugar de respetar los procesos autonómicos, el gobierno intenta cooptar nuestras organizaciones a través de programas asistenciales, y cuando no lo logra, utiliza la represión y la persecución selectiva para acabar la lucha.

A pesar de esas políticas contra nuestros pueblos los casos de Huehuetla, Puebla, Sulja, Guerrero, Unión Hidalgo, San Pedro Yosotato, San Isidro Aloapam, Oaxaca, y el pueblo púrhepecha, Michoacán, demuestran que la resistencia indígena continúa.

Saludamos desde la Sierra Norte de Puebla, territorio totonaco, a los Caracoles y a las Juntas de Buen Gobierno ${ }^{1}$, ya que representan la voz y el camino que estamos construyendo para nuestros pueblos.

Por todo lo anterior hacemos el siguiente PRONUNCIAMIENTO:

- Todo proyecto realizado en territorios indígenas debe contar invariablemente con el consentimiento de éstos y especificar los beneficios que obtendrán.

- Rechazamos la introducción y uso de las semillas y productos transgénicos en nuestras tierras y territorios.

- Rechazamos que se legisle en materia de medicina indígena tradicional hasta en tanto no exista un marco constitucional que reconozca los derechos colectivos de los pueblos indios.

-Rechazamos las políticas públicas indigenistas impulsadas por organizaciones gubernamentales.

- Rechazamos la explotación de los recursos naturales por empresas privadas. -Nos solidarizamos y hermanamos con el movimiento indígena de Latinoamérica, en especial con los hermanos de Ecuador y de Bolivia, en

\footnotetext{
${ }^{1}$ Caracoles y Juntas de Buen Gobierno, operan en territorio zapatista, en Chiapas.
} 
contra de los proyectos privatizadores y los planes de desarrollo globales como el Plan Panamá, ALCA, TLC y el corredor biológico mesoamericano. -Ante el anuncio hecho por PEMEX de que contratistas privados exploraran y explotaran más de 13 mil pozos en lo que se conoce como el PALEOCANAL DE CHICONTEPEC en la región de la huasteca, que se exija información y se rechace cualquier proyecto no consultado con los pueblos de la región.

-Rechazamos las políticas agrarias del gobierno que enfrentan a nuestras comunidades, por lo que exigimos el reconocimiento de la totalidad de los 412 comuneros de San Isidro Aloapam, Ixtlán, Oaxaca; el desalojo de los invasores del ejido de San Pedro Yosotato, Oaxaca, castigo a los autores intelectuales de la invasión, reparación de daños y garantizar la seguridad para los ejidatarios y sus familias. Así como la restitución de Contreras en el D.F., con base en los Títulos primordiales de 1563.

.Exigimos el respeto de las zonas arqueológicas de nuestro país, como templos sagrados de nuestros antepasados y que no se utilicen como zonas turísticas de lucro.

-Exigimos el cumplimiento de los Acuerdos de San Andrés y de la ley COCOPA.

Hacemos un enérgico llamado a los gobiernos estatales y federales para detener la opresión y violencia en nuestras comunidades:

.Exigimos el esclarecimiento e investigación de los asesinatos cometidos en contra de luchadores sociales y defensores de los derechos humanos en los casos de: Griselda Teresa Tirado Evangelio, asesora de la Organización Independiente Totonaca perpetrado el 6 de agosto del 2003 en Huehuetla, Puebla; el asesinato de Estela Ambrosio Luna miembro de la CEPCO asesinada el 5 de octubre del 2003 en San Agustín Loxicha, OAXACA; y, el asesinato del Dip. Carlos Sánchez López de la COCEI, abogado defensor de los presos de Unión Hidalgo, perpetrado el 17 de agosto del 2003 en Juchitán, Oaxaca.

-Exigimos alto a la tortura psicológica y las amenazas de muerte infringidas por el gobernador de Oaxaca José Murat contra Raúl Gatica del Consejo Indígena Popular de Oaxaca-Ricardo Flores Magón.

-Las organizaciones participantes en el Encuentro de naciones indígenas de México nos pronunciamos en contra de la estrategia gubernamental de represión y persecución contra los pueblos, comunidades, organizaciones y luchadores que defienden los derechos de los pueblos indios, por lo que exigimos la libertad incondicional de los indígenas presos en las diferentes cárceles de nuestro país.

-Libertad inmediata de Carlos Manso y Luis Alberto Marín, miembros del Consejo Ciudadano Unihidalguense y los presos de la Guevea de Humboldt, presos en el penal de Tehuantepec; la libertad de los presos políticos de los Loxichas, Teojomulco, CODECI y Oliverio Neri, presos en el penal de Ixcotel, Oaxaca. Libertad a los presos zapatistas de las cárceles de Chiapas y del país. 
En 2005 la UNITONA publicó una especie de manual para difundir los "derechos indígenas", e instruir sobre los mismos. Se trata de precioso material, que sirve como herramienta para consolidar la conciencia crítica de los indígenas. ${ }^{2}$

\section{Juzgado Indígena}

Fruto de la lucha indígena, resultado de la cuarta línea de acción de la UNITONA, en el año 2003, fue creado el Juzgado Indígena de Huehuetla, teniendo como misión "impartir justicia a la manera tradicional, mediante usos y costumbres”. Este juzgado fue reconocido por el Tribunal Superior de Justicia del Estado de Puebla y a su juez don Manuel Aquino. Camerino Aparicio, asesor de la UNITONA, considera el juzgado como una conquista, "pero allí no termina nuestra lucha por la autonomía” (Hernández, 2012, p. 196) -agrega-. El juzgado funciona con dos principios primordiales: la reparación del daño y la conciliación. También el juez indígena actúa como defensor del pueblo, basándose en una interpretación del Convenio 169 de Organización Internacional del Trabajo y los propios Acuerdos de San Andrés.

\section{Organización de las mujeres}

La presencia de las mujeres en todas estas luchas por la autonomía, ha sido muy importante. Como precioso ejemplo está la organización Corazón Floreciente de la Verdad (COFLOVER), adherida a las UNITONA, que nació en Tepango de Rodríguez, con el objeto de luchar contra el machismo. En esta organización ha sido muy importante la labor de la Congregación de Misioneras Guadalupanas del Espíritu Santo. Además de su trabajo rescatando los derechos de las mujeres, la COFLOVER se ha organizado para producir maíz, papa y frijol en común. También esta organización se ha dado a la tarea de recuperar rituales que estaban olvidados en Tepango, como la Danza de las Miguelitas o Danza de las Angelitas de Dios. Y tratando que esas danzas no se mercantilicen.

\section{Resistencia a los megaproyectos por comunidades indígenas de totonacos y nahuas}

\footnotetext{
${ }^{2}$ Derechos Indígenas, Unidad Indígena Totonaca Náhuatl, auspiciado por Zona Pastoral Norte, Arquidiócesis de
} Puebla. Lo presenta el vicario episcopal, padre Mario Pérez Pérez. 
En esta lucha por los derechos indígenas en la Sierra Norte de Puebla, se incluye la resistencia a megaproyectos. La UNITONA y otras organizaciones están presentes. Veamos algunas muestras de ello.

El Consejo por la Defensa de la Vida y de nuestro Territorio, Tiyat-Tlali -"tierra" o "territorio", en las lenguas totonaca y náhuatl, respectivamente-, integrado por varias organizaciones indígenas ${ }^{3}$, envió un comunicado informativo a la Coordinación Nacional de Enlace de Agentes de Pastoral y Pueblos Indígenas (EAPPI), para su conocimiento y apoyo. Damos a conocer los primeros párrafos que muestran las agresiones a las comunidades en sus derechos y el ánimo y actitud de éstas para resistir; el comunicado es de 1 de febrero de 2013, y está dirigido a la Conferencia del Episcopado Mexicano:

Gracia y paz en el Señor.

Estamos en un momento en el que se evidencia la fuerza con que se está imponiendo el proyecto neoliberal o capitalista en el mundo, y nosotros estamos asistiendo a un momento de voracidad de quienes tienen el poder económico para imponer un esquema a todo el mundo, una globalización que estamos viviendo, despojando a los demás de sus bienes, concentrando la riqueza en poquísimas manos. Esto no es un desarrollo donde entremos todos, sino un desarrollo desigual que implica el despojo. Están llegando nuevas empresas trasnacionales en nuestro país, como las mineras, las hidroeléctricas, etc., que quieren quedarse con nuestra riqueza, con la ley o sin la ley, ellos se quieren imponer, por las que estamos luchando por todos lados.

Es claro que los gobiernos están apoyando este modelo al crear y reformar leyes de nuestra Constitución que han facilitado a las empresas trasnacionales su implantación y su negocio entre nosotros; esto implica darles facilidades en todo sentido, para que se establezcan.

Esta es la razón por la cual les enviamos este comunicado, porque hemos vivido momentos muy especiales y difíciles en nuestras comunidades indígenas de este rincón de nuestro país, la Sierra Norte del Estado de Puebla, especialmente en la comunidad de Zaragoza, perteneciente al Municipio de Olintla, que sufre ataques y violaciones al derecho sobre su territorio, por parte de sus autoridades.

Se ha visto de manera clara y descaradamente la violación de nuestros derechos como pueblos y comunidades indígenas de totonacos y nahuas, al tomar decisiones para imponer estos proyectos de muerte, como la minería y la hidroeléctrica; sin consultarnos, ni decirnos la verdad de los efectos que trae estos proyectos; las decisiones los toman los gobiernos (federal, estatal y municipal) y las empresas privadas.

Nuestros asesores y especialistas se han dedicado a informarnos sobre los efectos negativos y pocos positivos que trae como consecuencia de estos proyectos, los mismos ingenieros de la empresa hidroeléctrica comexhidro, les hemos escuchado su palabra, y a partir de esto, las mismas comunidades indígenas, al conocer realmente los impactos que pueden acarrear, hemos tomado la decisión de rechazar estos proyectos.

\footnotetext{
${ }^{3}$ El Consejo Tiyat-Tlali está integrado por UNITONA (Unidad Indígena Totonaca - Náhuat), Pastoral Social de la Zona Norte de la Arquidiócesis de Puebla, Unitierra en Puebla, IMAEC, Timomachtikan, Unión de Cooperativas Tozepan Titoniske, CESDER, Centro de Estudios Ecuménicos, COPEVI, Nakuskgoy, Asociación Tetela hacia el futuro, CORDESI, Organización Indígena de Azan de Bienvenido.
} 
Sin embargo, esta defensa de nuestros territorios, nos han acarreado problemas fuertes y graves, han violentado nuestros derechos y obligado a vender nuestras tierras con amenazas; para ello, queremos relatarles de lo acontecido el pasado sábado 26 y domingo 27 de enero del año en curso, para que se solidaricen y animen a nuestros hermanos indígenas totonacos de Zaragoza. $^{4}$

El Consejo por la Defensa de la Vida y de Nuestro Territorio, Tiyat-Tlali, por los días que corren, continua su lucha por los derechos territoriales indígenas en la Sierra Norte de Puebla, especialmente resistiendo las acciones de las mineras. Natalia Rodríguez dice que es una "red de organizaciones sociales andando." (Rodriguez, Op. Cit., p. 90). Por convocatoria del sacerdote Mario Pérez Pérez, la reunión inaugural de esta gran red de organizaciones fue en agosto de 2012 en Cuetzalan del Progreso, en la casa parroquial de la Iglesia de los Jarritos; el propio padre Pérez propuso el nombre de Tiyat-Tlali, nombre en idiomas totonaco y náhuatl, como se dijo. (Rodriguez, Op. Cit., p. 91).

\section{Para terminar}

Como expresamos al comienzo de este texto, en los procesos de lucha de los pueblos indígenas de la Sierra Norte de Puebla hay un uso doble del Derecho; el que producen por medio de sus asambleas y sus prácticas comunitarias y las normas que dicta el Estado que les reconoce sus derechos; lo intentan hacer valer. Con relación a este último cito las palabras de un "Defensor del Territorio", que el 2 de diciembre de 2019 dijo en Olinta:

\footnotetext{
-Nos preguntamos sobre por qué ahora los pueblos vamos, o argumentamos a partir del derecho. Pues efectivamente porque quizás antes, no había esta posibilidad. Nosotros mismos, y nuestros derechos, estábamos en las catacumbas mismas de este Estado ¿no? En ningún momento se nos veía, en ningún momento se nos escuchaba, pese a que ya no estamos en la época de la colonia, en la que tampoco se nos escuchaba, ni se nos veía. Yo creo que fue un parteaguas muy importante el levantamiento zapatista.

El derecho dice que el primero en tiempo, es primero en derecho, pero no había derechos para nosotros. Y otra cuestión es que no había dónde sustentar nuestros derechos. Hasta que también empezamos a descubrir que hay unos convenios internacionales que desde hace mucho tiempo el gobierno firmó y que dijo que hay derechos de los pueblos indígenas. Entonces, empieza a verse, a vincularse, pero también, el otro problema es que no tenemos mucha gente en los pueblos que trate ese tema. Entonces, también esos elementos han jugado ¿no? Para que nuestros derechos como pueblos originarios, pues, no hayan llegado a las cortes, y lo otro también es la desconfianza, pues, si así nos tratan en nuestros espacios, en nuestros lugares. Si vamos al ministerio público, vamos con el juez, y no nos hacen caso, y nos ningunean, nos excluyen, pues, ese es otro elemento, la desconfianza. ${ }^{5}$
}

\footnotetext{
${ }^{4}$ Este documento lo hizo circular el padre Francisco Gabriel.

${ }^{5}$ Citado por Rodríguez, Op. Cit., p. 155.
} 
Aguascalientes, México, diciembre de 2020.

\section{Bibliografía:}

Hernández García, Milton Gabriel, Historia contemporánea del movimiento indígena en la Sierra Norte de Puebla, Ediciones Navarra y Centro de Investigaciones y Capacitación Rural, A.C. (CEDICAR), México, 2012.

Rodríguez, Natalia Micaela, "La experiencia de defensa del territorio del pueblo totonaca de la Sierra Norte de Puebla frente al proyecto hidroeléctrico Puebla 1", tesis para obtener el grado de maestra en derechos humanos, presentada en la Maestría en Derechos Humanos de la Universidad Autónoma de San Luis Potosí, 26 de agosto de 2020.

Tiyat-Tlali Consejo por la Defensa de la Vida y el Territorio, "Comunicado a la Conferencia del Episcopado Mexicano", Sierra Norte de Puebla, 1 de febrero de 2013.

Unidad Indígena Totonaca-Náhuatl, Derechos Indígenas, Arquidiócesis de Puebla, Zona Pastoral Norte.

Data de recebimento: 11.12 .2020

Data de aprovação: 15.02.2021 